\title{
SimUlation For Robotic STEREOTACTIC NEUROSURGERY
}

\author{
Josip Vidaković, Bojan Jerbić, Filip Šuligoj, Marko Švaco, Bojan Šekoranja
}
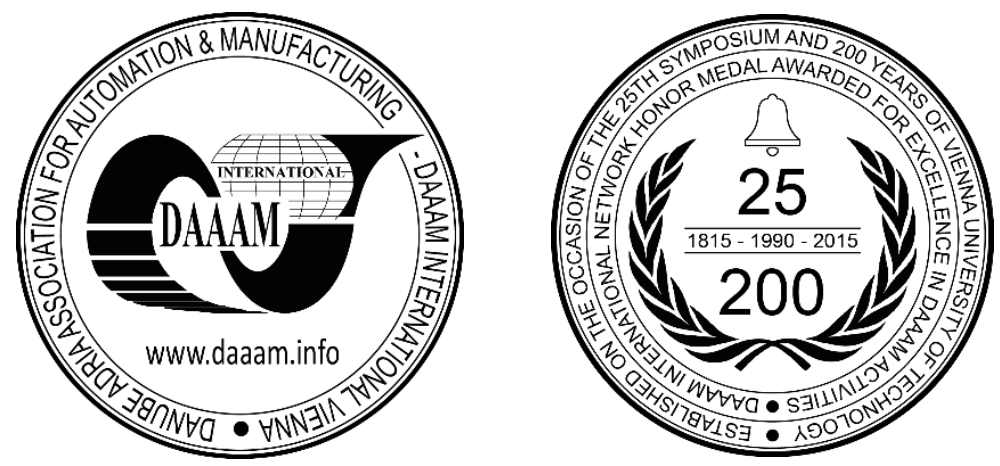

This Publication has to be referred as: Vidakovic, J[osip]; Jerbic, B[ojan]; Suligoj, F[ilip]; Svaco, M[arko] \& Sekoranja, B[ojan] (2016). Simulation for Robotic Stereotactic Neurosurgery, Proceedings of the 27th DAAAM International Symposium, pp.0562-0568, B. Katalinic (Ed.), Published by DAAAM International, ISBN 978-3-90273408-2, ISSN 1726-9679, Vienna, Austria

DOI: $10.2507 / 27$ th.daaam.proceedings.083

\begin{abstract}
Through the development of different research and commercial systems, robotic neurosurgery slowly becomes more standard in the medical field. Beyond other advantages, the possibility of frameless neurosurgery is an important reason for their consideration. However, the use of robots in these applications brings problems regarding robot-patient positioning and possible influence of kinematic restrictions during the execution of a surgical plan. To cope with these, a simulation concept for the control of critical positioning and kinematic parameters is presented in this paper. An optical tracking system, simulation software and stereotactic planning mechanism are integrated into one functional unit. It is a simulation tool which is intended to be used shortly after the operation scene is set in the operating room to ensure seamless operation flow. The system is successfully implemented and evaluated in real clinical use.
\end{abstract}

Keywords: Robotic surgery; Operation simulation; Operation planning; Stereotactic surgery

\section{Introduction}

Applications of robots in the medical field require a degree of absolute certainty when performing operation tasks. In neurosurgery, this represents a crucial factor for considering the use of modern robotic technology instead or in combination with standard neurosurgical equipment [1]. Generally, there are three main types of neurosurgical systems according to user interaction: automated, telesurgical and systems with shared control [2]. Because of their large dimensions and heavy built, telesurgical systems like the DaVinci system are not flexible in terms of spatial positioning. This results in their ability to operate just in a relatively small predetermined workspace in the operating room. On the other hand, automated systems which are often implemented as light-weight robot arms mounted on mobile platforms, are much more flexible. They can be configured to work in any workspace in the operating room and allow variable robot placement in respect to the patient. This makes them particularly suitable for surgical applications where the position and orientation of the patient can't be identical for every operation case.

In order to satisfy safety requirements in the operating room, technical reliability is one of the main aspects that robotical systems have to guarantee. The other one is functionality, which represents successful utilization of such equipment in individual operation cases. Robotic neurosurgical systems and robotic surgical systems in general have to deal with a high level of potential functional problems. They occur due to the unique parameters of every individual operation case (operation plan, patient position and orientation, collision with the environment). This is especially 
pronounced with flexible automated systems. Therefore, different planning strategies are developed for such systems with the purpose of solving or minimizing these problems. Here, some of the approaches will be mentioned. In [3], a positioning strategy is shown, which makes use of a workspace analysis for setting an optimization problem for every laparoscopic procedure performed by the laparoscopic robot individually. Another method for planning minimally invasive surgery that optimizes the relative position between the robot, patient and the operating room surrounding is presented in [4]. Other approaches are concerned with the dexterity of the robot actuator. Optimal robot workspaces are determined with the use of a dexterity criterion [5]. This way, a high operation completion rate can be guaranteed for all procedures that are carried out in a certain dexterous workspace.

A typical automated application in neurosurgery is frameless stereotactic surgery [6]. Advantages of robot kinematics are used for precise navigation of surgical instruments in the intracranial space of the patient [7]. The prerequisite for this kind of operation is the creation of a surgical plan. In the planning phase, the surgeon specifies points of interest as entry and target points in a CT or MR representation of the patient. These points are then transformed into the referent coordinate system for the robot. In the intraoperative phase, the robot localizes some referent coordinate system and performs positioning according to previously defined entry and target points which form unique trajectories. The position and orientation of trajectories are defined for every operation procedure according to the needs and specific demands of the patient's case.

The main challenge for the robot is to perform any of the defined trajectories regarding its relative position to the patient. The robot's ability to execute specific planned procedures is determined by the main three parameters: operation trajectories, patient position and robot base position. Here, robot-patient position and robot dexterity are the most important factors for successful operation completion.

Regardless which operation planning strategy is used, successful operation completion has to be guaranteed. For this, a concept for intraoperative procedure validation will be presented. The presented approach is not concerned with providing a strategy for optimal robot placement but giving a tool for final position validation of a stereotactic robot, which can guarantee successful operation completion. It is used at the very beginning of the operation, immediately after the patient and robot positioning is performed. The research is conducted in the scope of the project RONNA (Robotic Neuronavigation) [8].

The system originally consist of a dual arm robot configuration where one robot is responsible for the precise guidance of surgical tools and the second for assisting in different operation tasks (e.g. surgical drilling, biopsy needle/DBS electrode insertion). In the basic version, which will be in the scope of the application presented in this paper, the system is used for stereotactic procedures. This setup of the system consists of one general purpose industrial robot arm and two stereovision systems. One of the vision systems has a relatively wide working volume used for the global localization of the patient. The other one is a custom designed stereovision system mounted on the robot flange which uses a virtual TCP for the final, more accurate localization [9].

\section{Materials and methods}

The following system for intraoperative setup control is developed for the purposes of the RONNA project. It is composed of a NDI Polaris Spectra optical tracking system (OTS) and central simulation software. The OTS tracks passive retroreflective markers in its workspace volume and is able to give their precise position and orientation information. The system allows tracking of custom markers and is very commonly used in medical applications. In the simulation software, a simplified virtual setup of the operation environment is modelled using static CAD models of objects and instruments present in the operating room. These will be later used for collision detection. Also, a 3D model of the used robot with its corresponding kinematics is incorporated in the environment.

The system is designed to work in five steps:

1. Operation plan is loaded into the simulation software.

2. The OTS registers the position of the robot and the patient.

3. Corresponding virtual scene is set in the simulation environment.

4. The operation is simulated with exact robot movements.

5. Simulation results are evaluated and displayed to the operator. 
REAL SETUP
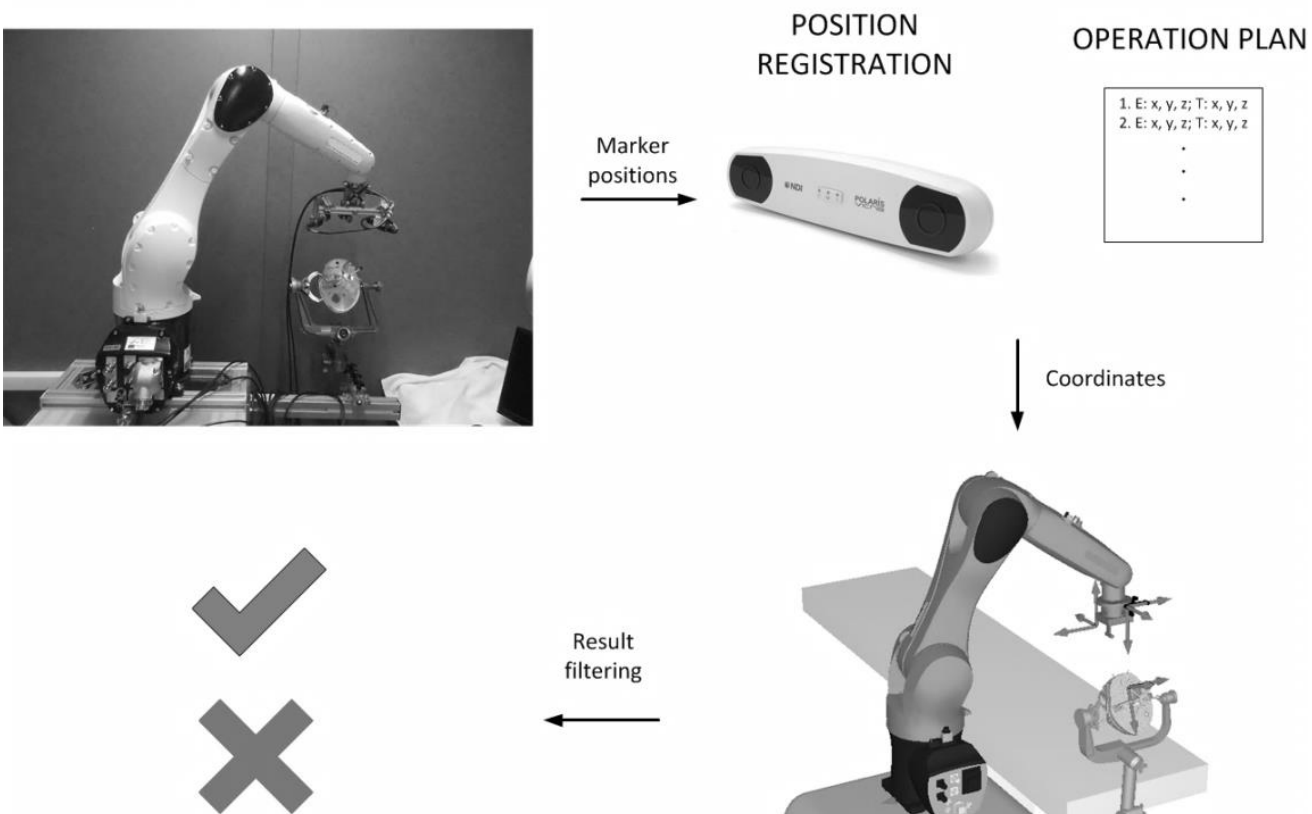

SIMULATION RESULTS
Result filtering
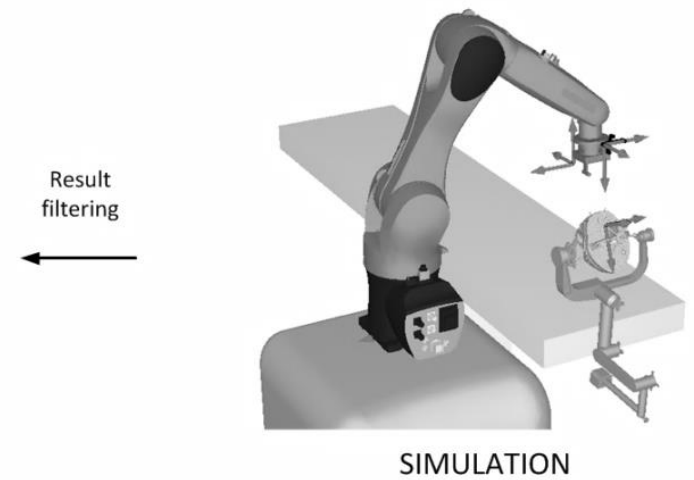

Fig. 1. Intraoperative simulation concept

\subsection{Position registration}

For the registration of the patient's position and orientation in the robot base frame, the system uses a fix passive retroreflective marker attached to the robot flange and a removable marker attached to a bone screw previously mounted on the patient's skull.

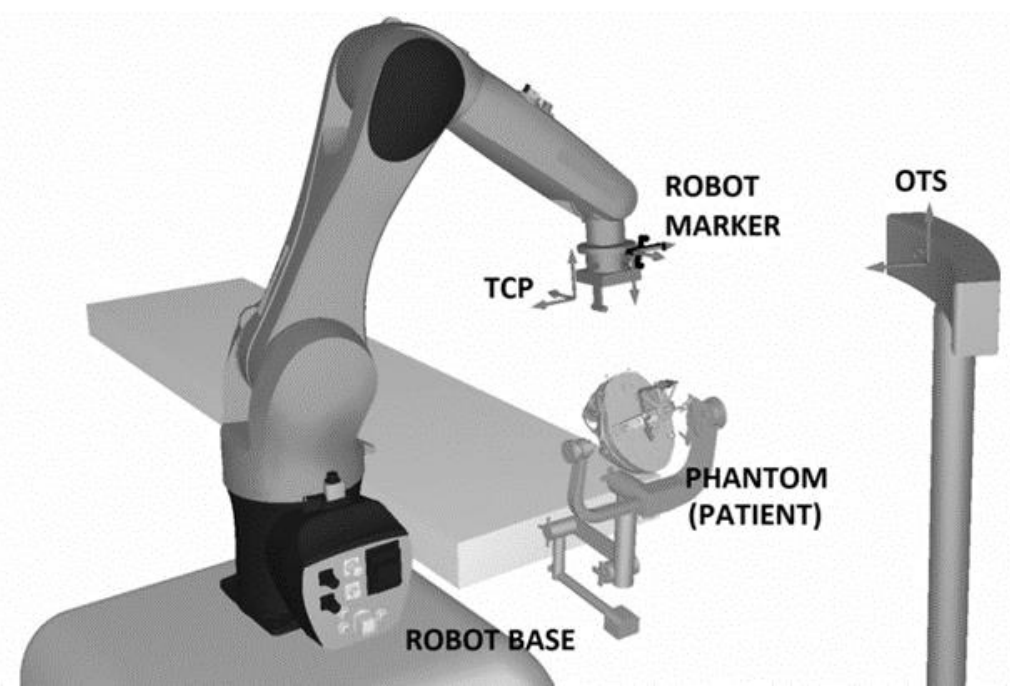

Fig. 2. Simulation setup

Using tracking data from the OTS, the system calculates the position of the patient in respect to the robot base coordinate system with the following homogenous transformations:

$$
\begin{aligned}
& T_{P A T I E N T}^{R O B O T_{1} B A S E}=T_{T C P}^{R O B O T_{-} B A S E} \times T_{P A T I E N T}^{T C P} \\
& T_{P A T I E N T}^{T C P}=T_{R O B O T \_M A R K E R}^{T C P} \times T_{P A T I E N T}^{\text {ROBOTAMARER }}
\end{aligned}
$$

The result of these transformations is a transformation matrix which contains the position and orientation of the patient marker. This is than sent to the simulation software via a TCP socket interface. 


\subsection{Operation plan}

The operation plan is created in medical imaging diagnostics software. Points of interest are marked by the surgeon in the form of entry and target points which form spatial trajectories. The trajectories are specifically chosen not to interfere with vital brain structures. Depending on this and the patient's diagnostics, every operation demands specific operation plans. Those points are transformed into the coordinate system of the patient and saved in a form suitable which will later serve as input for the robot in the operation procedure.

\subsection{Simulation execution and result filtering}

Based on the registered patient and the operation plan, the specific operation case can be simulated. The simulated robot performs the movement in two operational phases: localization and operation. In the first one, the robot moves its TCP into the center of every of three spheres, that the marker attached to the patient consists of, while maintaining a constant orientation. In the operation step, the robot positions its tool frame into the target of every planned trajectory with the orientation defined by the two points that make the trajectory (line in Cartesian coordinate system).

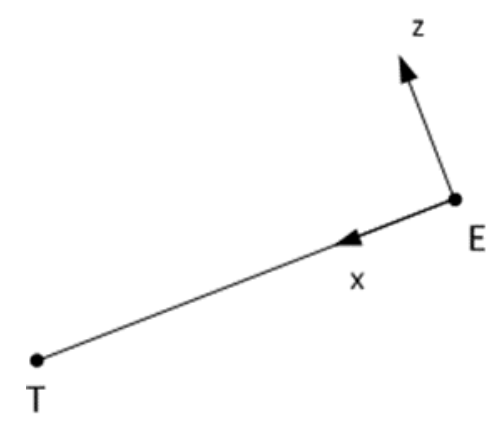

Fig. 3. Trajectory coordinate system orientation in respect to entry and target points

Orientations of trajectory frames are calculated from the two points (entry, target) and one supplementary point which is always vertical in respect to the origin of the coordinate system which is the entry point. The calculation is conducted as shown in (3).

$$
\begin{aligned}
& \vec{O}=\left[\begin{array}{lll}
X_{E} & Y_{E} & Z_{E}
\end{array}\right] \rightarrow \vec{O}=\vec{O}-\vec{O} \\
& \vec{X}=\left[\begin{array}{lll}
X_{T} & Y_{T} & Z_{T}
\end{array}\right] \rightarrow \vec{X}=\vec{X}-\vec{O} \\
& \overrightarrow{P x Z}=\left[\begin{array}{lll}
X_{E} & Y_{E} & Z_{E}+100
\end{array}\right] \rightarrow \overrightarrow{P x Z}=\overrightarrow{P x z}-\vec{O} \\
& \vec{X}=\frac{\vec{X}}{\|\vec{X}\|} ; \overrightarrow{P x Z}=\frac{\overrightarrow{P x z}}{\|\vec{X}\|} ; \vec{Y}=\frac{\vec{X} \times \overrightarrow{P x Z}}{\|\vec{X} \times \overrightarrow{P x Z}\|} \\
& \vec{Z}=\vec{Y} \times \vec{X}
\end{aligned}
$$

The homogenous rotation matrix is constructed as follows: $R=\left[\begin{array}{ccc}X[0] & Y[0] & Z[0] \\ X[1] & Y[1] & Z[1] \\ X[2] & Y[2] & Z[2]\end{array}\right]$.

Also, for the simulation of a real stereotactic procedure, the robot is then moved from the patient along the trajectory while maintaining the same orientation. In the simulation software, the robot's inverse kinematic problem is solved for every point on the trajectory $(p, g)$. One of the requirements to this operation is that the robot must not change its configuration in any step of the movement.

$$
J_{I K(p, g)}=\left[\begin{array}{llllll}
J 1 & J 2 & J 3 & J 4 & J 5 & J 6
\end{array}\right] \in \text { e.g.front,nonflip, elbowup }
$$

During linear movement the solution is also tested for singularities.

$$
\operatorname{det}(\operatorname{Jacobian}(p, g))=0
$$

Collision detection during all of these movements is performed for the simplified case of the operation environment. This gives the information about every trajectory and potential problem regarding its execution. 
If any of the parameters is not satisfied in the simulation, setup parameters i.e. position parameters of the operation have to be changed in order to retry the simulation and successfully complete the operation from the system point of view. This means the need for robot position change when the simulation does not complete successfully.

\section{Experiments and Results}

In order to validate the described simulation algorithm, the obtained results are compared with a corresponding experimental setup for stereotactic surgery. The simulation setup validity is here considered in terms of robot positioning accuracy. Spatial position coordinates of trajectory points (entry and target) have been obtained in the robot base coordinate system of the simulated robot and compared with the coordinates read from the real robot. The test is conducted by placing a real phantom for stereotactic testing in different regions of the robot workspace and executing the stereotactic procedure. The obtained results are presented in Table 1 and interpreted by the Euclidian distance as the measure of difference between the simulation and experiment.

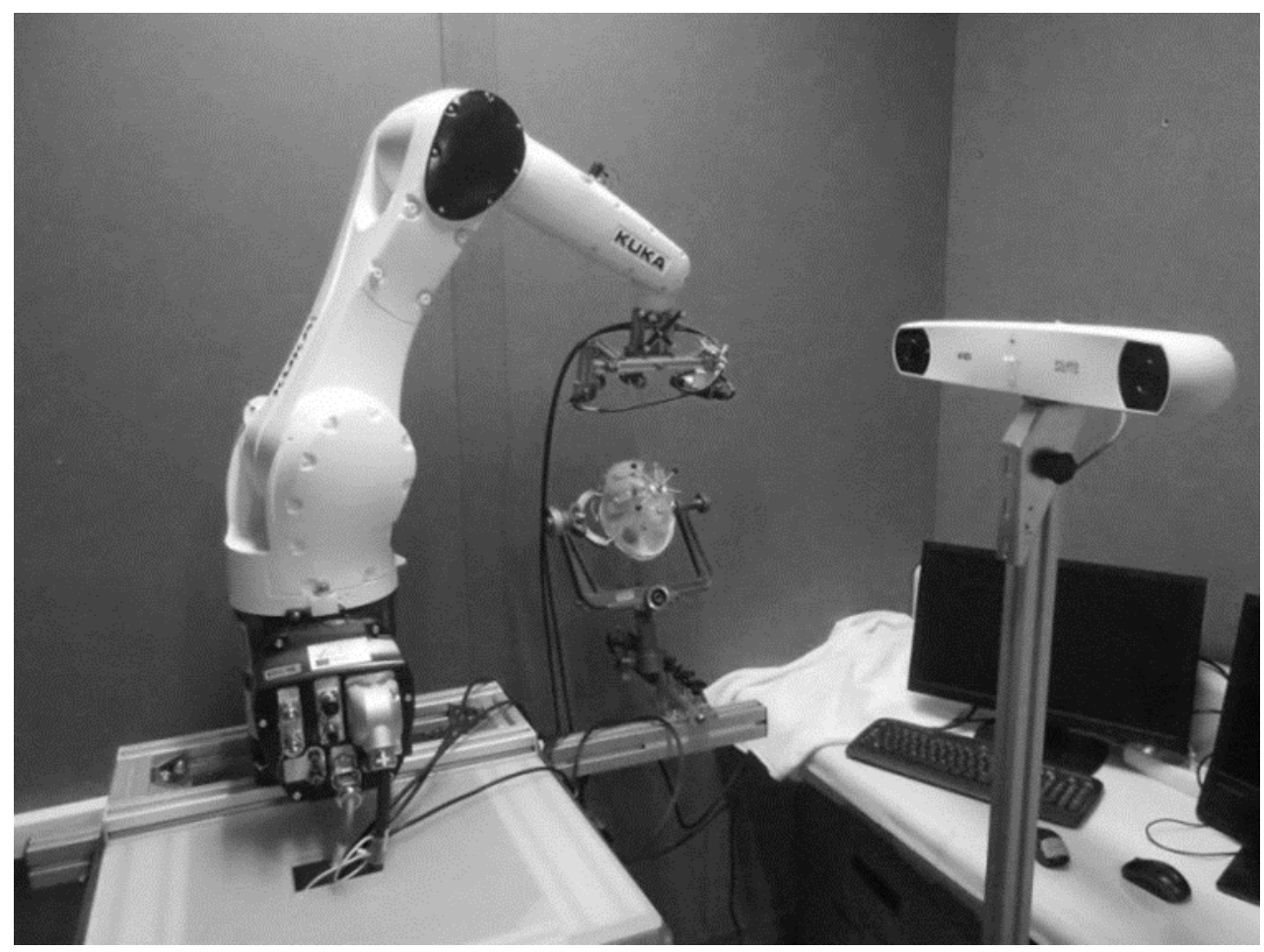

Fig. 4. Laboratory setup

\begin{tabular}{|c|c|c|c|}
\hline Robot base & $\begin{array}{c}\text { avg. error } \\
(\mathbf{m m})\end{array}$ & $\begin{array}{c}\text { min. error } \\
(\mathbf{m m})\end{array}$ & $\begin{array}{c}\text { max. error } \\
(\mathbf{m m})\end{array}$ \\
\hline X axis & 3.06 & 2.22 & 4.81 \\
\hline Y axis & 1.84 & 0.20 & 3.31 \\
\hline Z axis & 4.12 & 3.05 & 5.68 \\
\hline Euclidian distance & 5.45 & 3.77 & 8.14 \\
\hline
\end{tabular}

Table 1. Comparison of results

It is shown that the difference between the simulation and the real setup positioning data is in the range of 4 to $7 \mathrm{~mm}$, mostly depending on the position and orientation of the phantom i.e. patient marker. The error is present mainly due to inaccuracies of the OTS. These consist of general tracking errors and calibration inaccuracies of the OTS tool center point (TCP) obtained by a pivoting method. Robot calibration and positioning inaccuracies are here negligible because they are typically ranging under $1 \mathrm{~mm}$. However, for the relatively low number of 10 compared trajectories shown in the scope of this paper, and further 40-50 trajectories tested informal, the results indicate that this error does not rule out the simulation in terms of trustworthiness of the procedure completion success. In other words, the setup can reliably be used for intraoperative simulation as the last step and final control of the planning strategy, providing good insight for eventual problems in terms of kinematic restrictions and collision. 


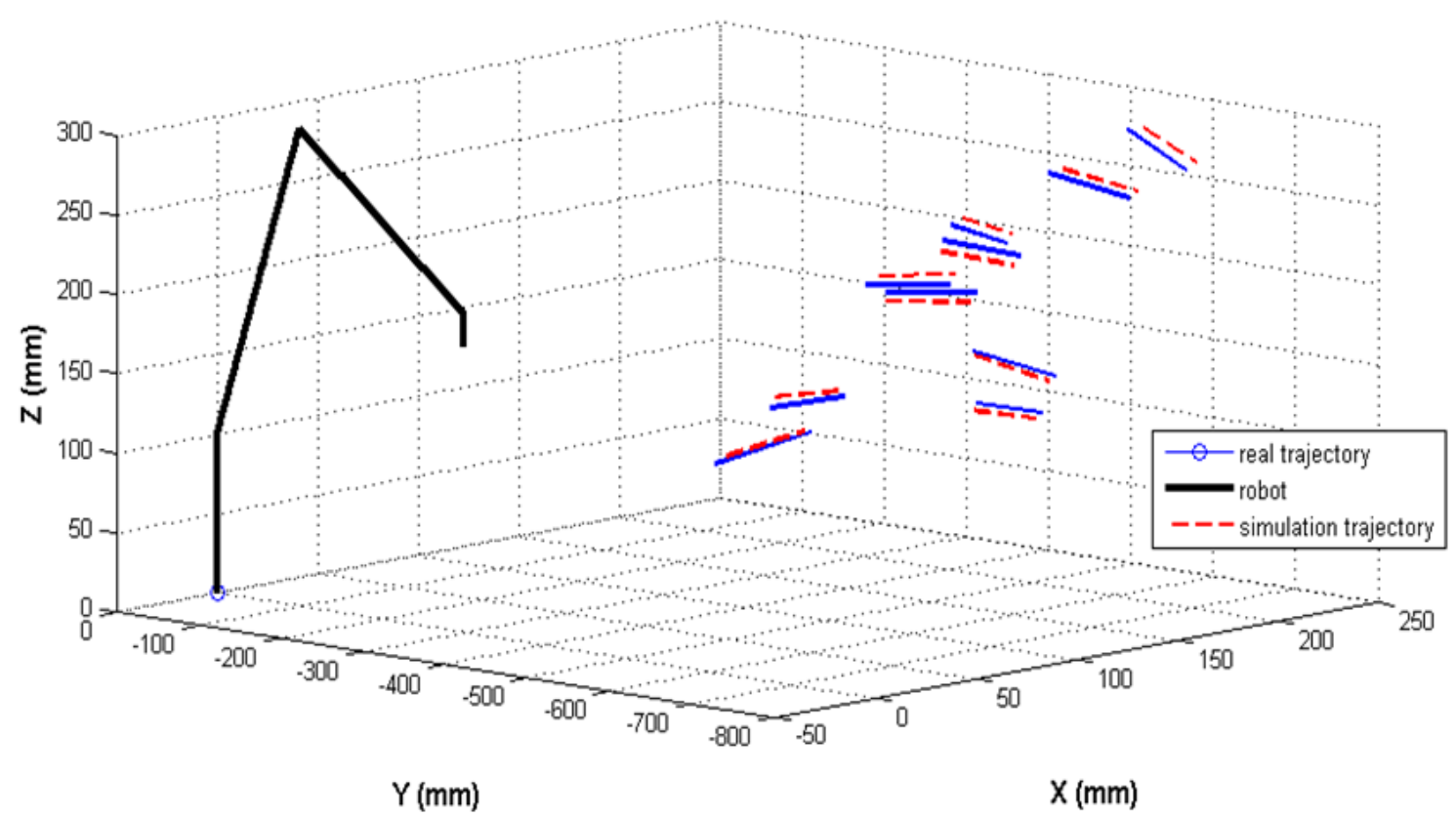

Fig. 5. Spatial comparison of 10 real and simulated trajectories

\section{Conclusion}

A simulation concept for the intraoperative setup control of a robotic system for neurosugical applications is presented in this paper. The simulation concept represents a simple and straightforward tool for introperative planning compatible with data and equipment already present in the standard medical environment (trajectories, CT models, Polaris OTS).

Robot kinematic constraints represent a crucial boundary when trying to satisfy the needs of spatial positioning of surgical tools in a surgical procedure. The presented approach eliminates eventual problems of this kind during the operation phase. This is done by simulating the procedure with real input parameters in its preparation phase, almost emediately after the patient is positioned in the operating room. The simulation can give all participants the insight into the operating procedure in a step-by-step way which can confirm the validity of the operational setup or emphasise problems regarding robot positioning or collisions. The approach is validated with the comparison of the real and simulated setup for different operation cases (plans).

This concept can be extended for surgical operations with a dual arm robot configuration that will be carried out in scope of the RONNA project. Also, it can be used for intraoperative control of various rmedical robotic applications which whose performans depends on spatial relations to the patient.

Future development will be directed toward developing a fully functional and automated preoprational planning strategy which will guarantee optimal setup configuration for the specific surgical operation plan before entering the surgical operating room. This could minimize the need for intraoperative simulation of this kind or be used along with it to satisfy strict medical safety standards.

\section{Acknowledgments}

Authors would like to acknowledge the support of the European Regional Development Fund through the project RONNA. Authors would also like to acknowledge the Croatian Scientific Foundation through the research project ACRON - A new concept of Applied Cognitive RObotics in clinical Neuroscience.

\section{References}

[1] J. D. Geiger and R. B. Hirschl. (2015). Innovation in surgical technology and techniques: Challenges and ethical issues, Semin. Pediatr. Surg., vol. 24, no. 3, pp. 115-121, Jun..

[2] N. Nathoo, M. C. Cavusoglu, M. A. Vogelbaum, and G. H. Barnett. (2005). In Touch with Robotics: Neurosurgery for the Future:, Neurosurgery, vol. 56, no. 3, pp. 421-433, Mar.

[3] B. Pan, Y. Fu, G. Niu, and D. Xu. (2013) Preoperative position planning strategy for laparoscopic robot, in Information and Automation (ICIA), IEEE International Conference on, pp. 1200-1205. 
[4] L. Adhami and E. Coste-Manirei. (2003). Optimal planning for minimally invasive surgical robots, IEEE Trans. Robot. Autom., vol. 19, no. 5, pp. 854-863, Oct.

[5] J. Liu, Y. Zhang, T. Wang, H. Xing, and Z. Tian. (2004). Neuromaster: a robot system for neurosurgery, in Robotics and Automation, 2004. Proceedings. ICRA’04. 2004 IEEE International Conference on, vol. 1, pp. 824-828.

[6] C. Faria, W. Erlhagen, M. Rito, E. De Momi, G. Ferrigno, and E. Bicho. (2015). Review of Robotic Technology for Stereotactic Neurosurgery, IEEE Rev. Biomed. Eng., vol. 8, pp. 125-137.

[7] T. A. Mattei, A. H. Rodriguez, D. Sambhara, and E. Mendel. (2014). Current state-of-the-art and future perspectives of robotic technology in neurosurgery, Neurosurg. Rev., vol. 37, no. 3, pp. 357-366, Jul.

[8] B. Jerbic, G. Nikolic, D. Chudy, M. Svaco, and B. Sekoranja. (2015). Robotic application in neurosurgery using intelligent visual and haptic interaction, Int. J. Simul. Model. IJSIMM, vol. 14, no. 1.

[9] M. Švaco, B. Šekoranja, F. Šuligoj, and B. Jerbić. (2014). Calibration of an Industrial Robot Using a Stereo Vision System, Procedia Eng., vol. 69, pp. 459-463. 